\title{
Evaluation of feeding efficiency and spillage in preterm infants during bottle and cup feeding: a randomized controlled trial
}

\begin{abstract}
Background: Establishing breastfeeding in very low birth weight (VLBW) infants remains a challenge in neonatal care. Bottle feeding is the most common supplement to breastfeeding in nurseries, followed by cup feeding. Spillage, however, can interfere with the accuracy of the volumes reported as consumed via cup feeding.

Objective: This study aimed to compare the feeding efficiency (as volume / minute) and the volume of milk spilled by VLBW infants during supplementary feeding by a bottle or a cup.

Methods: Participating infants weighed $<1500 \mathrm{~g}$ at birth, their gestational age ranged from 26 to 32 weeks. The volume of milk spilled during feeding was measured by weighing gauze napkins before and after the feed. The total volume of milk consumed was calculated as the volume offered minus the volume spilled.

Results: The methodology was applied in 23 preterm infants at the initiation of oral feeding, and the time of hospital discharge. The volume of milk consumed was higher when the supplementation was by bottle $(13.45 \mathrm{ml} \pm 9.8)$ than by cup $(4.22 \pm 3.4)$. There was no significant difference in the mean volume of milk spilled from bottles $(1.00 \pm 0.94)$ compared with cups $(0.64 \pm 0.72)$.

Conclusion: The volume of milk consumed from a cup is much lower than that from a bottle, and thus sufficient cup feeding will be more time-consuming than bottle feeding. Such a difference may be a negative factor to consider for NICU care, and it may explain the lack of acceptance of cup feeding by health professionals
\end{abstract}

\section{Well established}

In literature, there is no consensus about the feeding efficiency and milk spilled by VLBW infants during supplementary feeding by a bottle or a cup.

\section{Newly expressed}

This randomized crossover provided us information about the supplementary feeding. The volume of milk spilled was similar; however the bottle was more efficiency in feeding the VLBW infants and may be a negative factor to consider for NICU care.

\section{Background}

The mortality among preterm infants in developed countries has decreased dramatically in the last decade. The survival of very low-birth-weight (VLBW) infants has increased from $50 \%{ }^{1}$ to over $85 \%{ }^{2}$ The atypical early experience of VLBW infants affects their development and modifies their behavior. Establishing breastfeeding in preterm infants $(<37$ weeks gestation), especially those in the VLBW category, presents logistical issues for families and for the health-care staff, including identifying an appropriate enteral feeding method when the mother is not available to breastfeed. Bottle feeding is the most common supplementation to breastfeeding in nurseries, followed by cup feeding. ${ }^{3}$

However, the exposure of newborn infants to artificial nipples is strongly correlated with subsequent breastfeeding problems. ${ }^{4,5}$ These problems have frequently been attributed to a phenomenon termed "nipple confusion." Nipple confusion occurs when infants are exposed to two different feeding methods, the bottle and breast,
Volume 6 Issue 4 - 2017

\author{
Sabrina Lucena \\ Instituto Fernandes Figueira, Brazi
}

Correspondence: Sabrina Lucena, FIOCRUZ, Av. Rui Barbosa, Tel 552000000000,Email slucena@gmail.com

Received: March 16, 2017 | Published: March 27, 2017 resulting in a refusal to breastfeed. Consequently, cup feeding has been recommended over bottle feeding for the supplementation of term $^{6}$ as well as preterm infants. ${ }^{7,8}$

Cup feeding using a small cup without a lip is a recognized alternative method to feed breast milk to an infant ${ }^{9}$ and was established for feeding infants who could not be breastfed from birth. ${ }^{10}$ However, a Cochrane review ${ }^{11}$ concluded that, as a supplement to breastfeeding, cup feeding could not be recommended over bottle feeding because it had no significant benefit in maintaining breastfeeding beyond hospital discharge. Additionally, the review suggested that cup feeding had the potential to unacceptably lengthen the hospital stay.

There is a lack of studies on alternative methods of feeding infants. Some studies have shown that, in some cases, the use of bottles has no significant impact on breastfeeding, 7,12 and other studies have raised questions about the efficiency and efficacy of cup feeding. The time required to cup feed is often longer ${ }^{13}$ which impacts nursing time. Spillage, however, can interfere with the accuracy of the volumes reported as consumed via cup feeding. ${ }^{13-15}$

It is important to discuss the volume of milk lost by spillage due to a low intake could affect weight gain and consequently the length of stay of the newborn. ${ }^{7}$ A less efficient feeding practice will result in more time being spent on feeding. ${ }^{13,15}$ Such a difference may be a negative factor to consider for NICU care, and it may explain the lack of acceptance of cup feeding by health professionals. ${ }^{15}$

The objective of this study was to compare the feeding efficiency (as volume / minute) and the volume of milk spilled by VLBW infants during supplementary feeding by a bottle or a cup. 


\section{Methods}

Infants born at the National Institute of Women, Children and Adolescent Health Fernandes Figueira in Brazil from August 2009 to December 2012 were eligible. The inclusion criteria were the following: birth weight $<1500 \mathrm{~g}$, gestational age from 26 to 32 weeks, and an absence of congenital anomalies, severe asphyxia (as defined by 5 -min Apgar score $<5$ or convulsions in the first $24 \mathrm{~h}$ ), or bronchopulmonary dysplasia. Infants with sepsis or/and intraventricular hemorrhage grade III or IV (documented by ultrasound) were excluded. Informed consent was obtained from the parents or guardians of the patient prior to their inclusion. The study was approved by the Institutional Review Board of the National Institute of Women, Children and Adolescent Health Fernandes Figueira (study protocol 0059.0.008.000-06).

A randomized crossover design was used. Randomization occurred when study infants were deemed ready to begin oral feeding by their speech therapist and the medical team. Indicators of oral readiness in this unit include maintenance of physiologic stability during nonnutritive sucking, eagerness to suck, and sustained alertness.

The order of the alternative feeding methods used was determined by coin toss and comprised one cup feeding followed by one bottle feeding or one bottle feeding followed by one cup feeding. There was at least one gavage feeding between these two oral feedings. Study feedings were given when the infants were in conscious state 4 on the Brazelton scale ${ }^{16}$ (alertness active or inactive), had their diaper changed, and were hungry. The preterm infants were in a semi-sitting position. At discharge, the same study protocol was repeated.

The babies had each of their two study feedings administered by the same professional, who had been trained and had much experience in the techniques used. Infants were swaddled, with their head and neck supported in a semi-upright position. For both study feedings, the baby's movements, pulse oximetry and cardiac parameters were monitored to determine when to pause or terminate the feeding session.

Cup feedings were given with a medicine cup (by Medela $(\mathbb{B}$ ), using the procedure previously published. ${ }^{9}$ The rim of the cup, containing the prescribed amount of milk for the feed, was placed gently against the infant's lower lip. The cup was tipped so that milk just touched the lower lip; it was not poured into the infant's mouth. The cup was refilled as necessary. Bottle feeding was given from a graduated feeder with an orthodontic silicone nipple (by NUK ${ }^{\circledR}$ ).

Feedings were paused whenever an adverse physiological event occurred, such as sustained oxygen saturation below $85 \%$. The feedings were resumed after recovery from the event.

All infants were assessed during the first three minutes of feeding. During this period, the gauze napkins used were weighed before and after the feed, and the total amount of spillage was calculated. The total consumed volume was then calculated by deducting the spillage volume from the offered volume. The feeding efficiency was expressed as the intake of milk in relation to the time taken to feed.

Statistical analysis used Student's t-test for unpaired samples and was followed by testing for normality using the One-sample Kolmogorov-Smirnov test.

\section{Results}

The two feeding methods were applied to 23 preterm infants on two different occasions: at the start of the oral feeding and at hospital discharge. Demographic characteristics of the preterm infants are described in Table 1.
Table I Population characteristics. National Institute of Women, Children and Adolescent Health Fernandes Figueira, Rio de Janeiro, Brazil, 20I2. $(n=23)$

\begin{tabular}{lll}
\hline & Mean & SD \\
\hline Birth weight (g) & 1 I30,6 & $\pm 203,15$ \\
Gestational age at birth (weeks) & $303 / 7$ & $\pm I 6 / 7$ \\
Duration of O2 therapy (days) & 4,56 & $\pm 6,08$ \\
Duration of orotracheal tube use (days) & 0,84 & $\pm I, 72$ \\
Days of life until birth weight recovered & 12,46 & $\pm 6,0 \mathrm{I}$ \\
& Median & \\
APGAR I min. & 7 & \\
APGAR 5 min. & 9 & \\
Start of Oral Feeding & & \\
Days of Life & 31,84 & $\pm 8,8$ \\
Weight (g) & 1615,4 & $\pm 260,96$ \\
Gestacional Age (weeks) & $352 / 7$ & $\pm 44 / 7$ \\
Discharge & & \\
Days of Life & 44 & $\pm 8,72$ \\
Weight (g) & 1912,08 & $\pm 296,31$ \\
Gestacional Age (weeks) & $365 / 7$ & $\pm I 6 / 7$ \\
\hline
\end{tabular}

\section{Volume of milk consumed}

At the start of oral feeding, $92 \%$ of the newborns ingested more with the bottle and $8 \%$ of the newborns ingested more with the cup. The volume of milk consumed was higher with the use of the bottle $(13.45 \mathrm{ml} \pm 9.8)$ when compared with the cup $(4.22 \pm 3.4)$. Consequently, the feeding efficiency was significantly higher with the use of the bottle $(4.48 \mathrm{ml} / \mathrm{min})$ compared with the cup $(1.4 \mathrm{ml}$ / min). The differences between the bottle and cup efficiencies were statistically significant (Table 2). At discharge, we found the same pattern of response, as $100 \%$ of the newborns ingested more with the bottle than with the cup.

Table 2 Feeding efficiency and milk spillage in cup and bottle at the start of oral feeding and at discharge

\begin{tabular}{llll}
\hline Start Oral Feeding & Cup & Bottle & P* \\
\hline & Mean \pm SD & Mean \pm SD & \\
Milk consumed $(\mathrm{ml})$ & $4,22 \pm 3,4$ & $\mathrm{I} 3,45 \pm 9,8$ & $0,000(\mathrm{I})$ \\
Efficiency $(\mathrm{ml} / \mathrm{min})$ & $\mathrm{I}, 4 \pm \mathrm{I}, \mathrm{I}$ & $4,48 \pm 3,2$ & $0,000(\mathrm{I})$ \\
Spilling $(\mathrm{ml})$ & $0,64 \pm 0,72$ & $\mathrm{I}, 00 \pm 0,94$ & $0, \mathrm{I} 45(2)$ \\
Discharge & & & \\
Milk consumed $(\mathrm{ml})$ & $5,34 \pm 3,4$ & $\mathrm{I} 3,79 \pm 5,8$ & $0,000(\mathrm{I})$ \\
Efficiency $(\mathrm{ml} / \mathrm{min})$ & $\mathrm{I}, 78 \pm \mathrm{I}, \mathrm{I}$ & $4,59 \pm \mathrm{I}, 9$ & $0,000(\mathrm{I})$ \\
Spilling $(\mathrm{ml})$ & $0,67 \pm 0,77$ & $0,6 \pm \mathrm{I}, \mathrm{I} 6$ & $0,7 \mathrm{I} 5(2)$ \\
\hline
\end{tabular}

*I. Paired t-test

2.Wilcoxon signed-rank test

\section{The degree of spilling}

Comparing the volume of spilled milk during feeds offered by cup and by bottle, we found that, at start of oral feeding, 56\% of newborns had greater spilling during bottle-feeding and 36\% when the cup was used, and $8 \%$ of newborns had similar spilling from both. When evaluated at discharge, $40 \%$ of newborns had higher spilling during bottle-feeding and $60 \%$ spilled more when the cup was used. At the start of oral feeding, there was no significant difference in the mean of milk spilled using a cup $(0.64$ $\pm 0.72)$ compared to a bottle $(1.00 \pm 0.94)$. Similar results were obtained at discharge $(0.67 \pm 0.77 /$ cup; $0.6 \pm 1.16$ /bottle, Table 2). Preterm infants took part in a clinical trial where they were randomized to receive either cups or bottles during the hospital stay, minimizing this type of confounding. 


\section{Discussion}

The prolonged hospitalization VLBW newborns contributes to difficulties in maintaining the mother's milk production, increasing the likelihood of artificial feeding.

The bottle is the most common method used to supplement breastfeeding, followed by the cup. ${ }^{3}$

The technique used to offer milk is a very important to any discussion of milk intake, as it affects the newborn's opportunity to self-regulate the demand for milk, and some studies have suggested that the cup may have advantages over the bottle. ${ }^{17}$

We used the technique described by Lang, Lawrence and Orme (1994), ${ }^{9}$ currently recommended in the literature, in which the milk touches the lower lip of the newborn and is ingested through an anteroposterior movement of the tongue. However, one study ${ }^{14}$ that investigated the volume of milk ingested when offered by different methods gently poured milk from a cup into the infant's mouth, small amounts at a time. This pouring technique cannot efficiently assess the intake of milk because it will be biased by the examiner who administers the diet.

We observed that, the volume of milk consumed at the start of oral feeding was higher during bottle feeding than during cup feeding. The same result was observed at discharge, when the tests showed higher intake of milk during bottle-feeding. Marinelli, Burke and Dodd ${ }^{13}$ also observed a lower intake of milk when it was offered by cup; however, it is unclear how this volume was measured, and it does not take spilling during administration into account.

In contrast, the study by Malhotra et al., ${ }^{14}$ noted that the volume of milk consumed by cup was higher when compared to the bottle. Their data are questionable, however, because the technique used to offer the milk determines how much is poured into the child's mouth. This is not true of the bottle, from which the milk is extracted by suction.

The scarcity and the lack of methodological rigor of studies found in the literature makes comparisons with our data impossible.

Analyzing the spilling of milk during diet administration, we encountered a similar lack of studies. Malhotra et al., ${ }^{14}$ performed this analysis using a plastic bib to measure the spilling of milk and concluded that the loss of milk was higher in the cup compared to the bottle. However, their study may be criticized because it was not a controlled randomized trial. Similarly, Aloysius and Hickson, ${ }^{15}$ in prospective crossover study, also observed greater spilling of milk during the use of the Paladai (a cup type commonly used in India) compared with the bottle.

When evaluating the spilling of milk during a complementary diet through the use of cup and bottle, we found no significant differences. In our randomized crossover clinical trial design, each newborn serves as his own control. Our data are further strengthened because we are not comparing two populations but the same population by fed by two different methods.

We believe that, when the cup is administered in the right way, there is no significant spilling of milk, as the infant drinks according to his or her ability. This variable was controlled in our study because the cup was always administered by same evaluator (who had enough experience in the technique). Perhaps this accounts for the differences between our findings and those in the literature. ${ }^{15}$

Both power efficiency and the loss of milk are very dependent on the feeder, so this variable was also controlled in our study.
In clinical practice, the greater loss of milk that is expected from cup feeding is perceived as an obstacle to the parents' and health professionals' acceptance of cup use. Training is necessary for the effective use of the cup. Many mothers prefer to use the bottle to administer a supplement, and they report the greater spilling of milk, rather than the satiety of the newborn, the increased time spent on feeding, or the difficulty in administration, ${ }^{7}$ as the problem with cup feeding.

\section{Conclusion}

Although we did not observe differences in the volume of milk spilled, the volume of milk ingested by cup feeding is much lower when compared with bottle feeding. Cup feedings, in practice, demand longer feeding times: an aspect already covered in two prospective crossover studies..$^{13,15}$ This can be a disadvantage for NICU routines and may limit acceptance by health professionals. Moreover, cup feeding may have the potential to fatigue the baby, leading to a risk for aspiration, as well as creating time pressures on limited nursing resources..$^{15}$

\section{Acknowledgments}

None.

\section{Conflicts of interest}

Author declares there are no conflicts of interest.

\section{Funding}

None.

\section{References}

1. Stewart AL, Reynolds EOR, Lipscomb AP. Out- come for infants of very low birthweight: survey of world literature. Lancet. 1981; 1981 1(8228):1038-1040.

2. Horbar JD, Badger GJ, Lewit EM, et al. Hospital and patient characteristics associated with variations in 28-day mortality rates for very low birth weight infants. Vermont Oxford Network. Pediatrics. 1997;99(2):149-156.

3. Al-Sahab B, Feldman M, Macpherson A, et al. Which method of breastfeeding supplementation is best? The beliefs and practices of paediatricians and nurses. Paediatr Child Health . 2010;15(7):427-431.

4. Howard CR, Howard FM, Lanphear B, et al. Randomized clinical trial of pacifier use and bottle-feeding or cupfeeding and their effect on breastfeeding. Pediatrics. 2003;111(3):511-518.

5. Neifert M, Lawrence R, Seacat J. Nipple confusion: toward a formal definition. J Pediatr. 1995;126(6):S125-S129.

6. Brown SJ, Alexander J, Thomas P. Feeding outcome in breast-fed term babies supplemented by cup or bottle. Midwifery. 1999;15(2):92-96.

7. Collins CT, Ryan P, Crowther CA, et al. Effect of bottles, cups, and dummies on breast feeding in preterm infants: a randomised controlled trial. BMJ. 2004;329(7459):193-198.

8. Rocha NM, Martinez FE, Jorge SM. Cup or bottle for preterm infants: effects on oxygen saturation, weight gain, and breastfeeding. J Hum Lact .2002;18(2):132-138.

9. Lang S, Lawrence CJ, Orme RL. Cup feeding: an alternative method of infant feeding. Arch Dis Child. 1994;71(4):365-369.

10. Gupta A, Khanna K, Chattree S. Cup feeding: an alternative to bottle feeding in a neonatal intensive care unit. $J$ Trop Pediat. 1999;45(2):108-110. 
11. Flint A, New K, Davies MW. Cup feeding versus other forms of supplemental enteral feeding for newborn infants unable to fully breastfeed. Cochrane Database of Systematic Reviews Issue 2. Art. No: CD005092. 2007.

12. Schubiger G, Schwarz U, Tonz O. UNICEF/WHO Baby-Friendly Hospital Initiative: does the use of bottles and pacifiers in the neonatal nursery prevent successful breastfeeding? Eur $J$ Pediatric. 1997; 156(11):874-877.

13. Marinelli KA, Burke GS, Dodd VL. A comparison of the safety of cupfeedings and bottlefeedings in premature infants whose mothers intend to breastfeed. J Perinatol . 2001;21(6):350-355.
14. Malhotra N, Vishwambaran L, Sundaram KR, et al. A controlled trial of alternative methods of oral feeding in neonates. Early Human Development. 1999;54(1):29-38.

15. Aloysius A, Hickson M. Evaluation of paladai cup feeding in breastfed preterm infants compared with bottle feeding. Early Hum Dev. 2007;83(9):619-621.

16. Brazelton TB. Neonatal behavior evaluation scale. Neuropsychiatric Enfance Adolescent . 1983;31(2-3): 61-96.

17. Vallenas C, Savage F. Evidence for the Ten Steps to Successful Breastfeeding. Division of Child Health and Development, World Health Organization. 1998 